\title{
Positive solutions for a system of nonlinear
} fractional nonlocal boundary value problems with parameters and $p$-Laplacian operator

\author{
Xinan $\mathrm{HaO}^{1 *}$, Huaqing Wang ${ }^{1}$, Lishan $\mathrm{Liu}^{1,2}$ and Yujun Cui ${ }^{3}$
}

\section{"Correspondence:}

haoxinan2004@163.com

'School of Mathematical Sciences,

Qufu Normal University, Qufu,

Shandong 273165, P.R. China

Full list of author information is

available at the end of the article

\begin{abstract}
In this paper, we investigate the existence of positive solutions for a system of nonlinear fractional differential equations nonlocal boundary value problems with parameters and $p$-Laplacian operator. Under different combinations of superlinearity and sublinearity of the nonlinearities, various existence results for positive solutions are derived in terms of different values of parameters via the Guo-Krasnosel'skii fixed point theorem.
\end{abstract}

Keywords: positive solution; $p$-Laplacian operator; fractional differential system; nonlocal boundary value problem

\section{Introduction}

In this paper, we investigate the following system of nonlinear fractional differential equations nonlocal boundary value problems with parameters and $p$-Laplacian operator:

$$
\left\{\begin{array}{l}
-D_{0^{+}}^{\alpha_{1}}\left(\varphi_{p_{1}}\left(D_{0^{+}}^{\beta_{1}} u(t)\right)\right)=\lambda f(t, u(t), v(t)), \quad 0<t<1, \\
-D_{0^{+}}^{\alpha_{2}}\left(\varphi_{p_{2}}\left(D_{0^{+}}^{\beta_{2}} v(t)\right)\right)=\mu g(t, u(t), v(t)), \quad 0<t<1, \\
u(0)=u(1)=u^{\prime}(0)=u^{\prime}(1)=0, \quad D_{0^{+}}^{\beta_{1}} u(0)=0, \quad D_{0^{+}}^{\beta_{1}} u(1)=b_{1} D_{0^{+}}^{\beta_{1}} u\left(\eta_{1}\right), \\
v(0)=v(1)=v^{\prime}(0)=v^{\prime}(1)=0, \quad D_{0^{+}}^{\beta_{2}} v(0)=0, \quad D_{0^{+}}^{\beta_{2}} v(1)=b_{2} D_{0^{+}}^{\beta_{2}} v\left(\eta_{2}\right),
\end{array}\right.
$$

where $\alpha_{i} \in(1,2], \beta_{i} \in(3,4], D_{0^{+}}^{\alpha_{i}}$ and $D_{0^{+}}^{\beta_{i}}$ are the standard Riemann-Liouville derivatives, $\varphi_{p_{i}}(s)=|s|^{p_{i}-2} s, p_{i}>1, \varphi_{p_{i}}^{-1}=\varphi_{q_{i}}, \frac{1}{p_{i}}+\frac{1}{q_{i}}=1, \eta_{i} \in(0,1), b_{i} \in\left(0, \eta_{i}^{\frac{1-\alpha_{i}}{p_{i}-1}}\right), i=1,2 . f, g \in C([0,1] \times$ $\left.[0,+\infty)^{2},[0,+\infty)\right), \lambda$ and $\mu$ are positive parameters.

Fractional differential equation models are proved to be more adequate than integer order models for some problems in science and engineering. Fractional differential equations play a very important role in various fields due to their deep real world background. For an introduction of fractional calculus and fractional differential equations, we refer the reader to [1-3] and the references therein.

Turbulent flow in a porous medium is a fundamental mechanics problem. For studying this type of problem, Leibenson [4] introduced differential equations with $p$-Laplacian

(c) The Author(s) 2017. This article is distributed under the terms of the Creative Commons Attribution 4.0 International License (http://creativecommons.org/licenses/by/4.0/), which permits unrestricted use, distribution, and reproduction in any medium, provided you give appropriate credit to the original author(s) and the source, provide a link to the Creative Commons license, and indicate if changes were made. 
operator

$$
\left(\varphi_{p}\left(u^{\prime}(t)\right)\right)^{\prime}=f(t, u(t))
$$

The study of differential equation with $p$-Laplacian operator is of significance theoretically and practically. It is quite natural to study fractional differential equation relative to equation (1.2).

Recently, many scholars have paid more attention to the fractional order differential equation boundary value problems with $p$-Laplacian operator, see [5-23]. In [15], Lu et al. investigated a class of boundary value problems for fractional differential equations with $p$-Laplacian

$$
\left\{\begin{array}{l}
D_{0^{+}}^{\alpha}\left(\varphi_{p}\left(D_{0^{+}}^{\beta} u(t)\right)\right)=f(t, u(t)), \quad 0<t<1, \\
u(0)=u^{\prime}(0)=u^{\prime}(1)=0, \quad D_{0^{+}}^{\beta} u(0)=D_{0^{+}}^{\beta} u(1)=0
\end{array}\right.
$$

where $\alpha \in(1,2], \beta \in(2,3], D_{0^{+}}^{\alpha}$ and $D_{0^{+}}^{\beta}$ are the standard Riemann-Liouville derivatives, $f \in C([0,1] \times[0,+\infty),[0,+\infty))$. The existence and multiplicity results of positive solutions were obtained by using the Guo-Krasnosel'skii fixed point theorem, the Leggett-Williams fixed point theorem and the upper and lower solutions method. Xu and Dong [17] considered the following three point boundary value problem of fractional differential equation with $p$-Laplacian operator:

$$
\left\{\begin{array}{l}
-D_{0^{+}}^{\alpha}\left(\varphi_{p}\left(D_{0^{+}}^{\beta} u(t)\right)\right)=f(t, u(t)), \quad 0<t<1, \\
u(0)=u(1)=u^{\prime}(0)=u^{\prime}(1)=0, \quad D_{0^{+}}^{\beta} u(0)=0, \quad D_{0^{+}}^{\beta} u(1)=b D_{0^{+}}^{\beta} u(\eta),
\end{array}\right.
$$

where $\alpha \in(1,2], \beta \in(3,4], D_{0^{+}}^{\alpha}$ and $D_{0^{+}}^{\beta}$ are the standard Riemann-Liouville derivatives, $\eta \in(0,1), b \in\left(0, \eta^{\frac{1-\alpha}{p-1}}\right), f \in C([0,1] \times[0,+\infty),[0,+\infty))$. The existence and uniqueness of positive solutions were obtained by using the upper and lower solutions method and Schauder's fixed point theorem, the iterative sequences for the unique solution were also given.

In [18], Zhang et al. considered the eigenvalue problems of fractional differential equations with integral boundary conditions and $p$-Laplacian operator

$$
\left\{\begin{array}{l}
-D_{0^{+}}^{\alpha}\left(\varphi_{p}\left(D_{0^{+}}^{\beta} u(t)\right)\right)=\lambda f(t, u(t), \quad 0<t<1, \\
u(0)=0, \quad D_{0^{+}}^{\beta} u(0)=0, \quad u(1)=\int_{0}^{1} u(s) d A(s),
\end{array}\right.
$$

where $\alpha \in(0,1], \beta \in(1,2], D_{0^{+}}^{\alpha}$ and $D_{0^{+}}^{\beta}$ are the standard Riemann-Liouville derivatives, $A$ is a function of bounded variation, $\int_{0}^{1} u(s) d A(s)$ is the Riemann-Stieltjes integral, $f(t, u)$ : $(0,1) \times(0,+\infty) \rightarrow[0,+\infty)$ is a continuous function that may be singular at $t=0,1$ and $u=0$. The existence of positive solutions of problem (1.3) was established by using the upper and lower solutions method and Schauder's fixed point theorem. Lv [20] discussed an $m$-point boundary value problem of fractional differential equation with $p$-Laplacian operator

$$
\left\{\begin{array}{l}
D_{0^{+}}^{\alpha}\left(\varphi_{p}\left(D_{0^{+}}^{\beta} u(t)\right)\right)+\varphi_{p}(\lambda) f(t, u(t))=0, \quad 0<t<1, \\
u(0)=0, \quad D_{0^{+}}^{\gamma} u(1)=\sum_{i=1}^{m-2} \xi_{i} D_{0^{+}}^{\gamma} u\left(\eta_{i}\right), \quad D_{0^{+}}^{\beta} u(0)=0,
\end{array}\right.
$$


where $\alpha, \gamma \in(0,1], \beta \in(1,2], \beta-\alpha-1 \geq 0, \beta-\gamma-1 \geq 0, \lambda$ is a positive parameter, $0<$ $\xi_{i}, \eta_{i}<1, \sum_{i=1}^{m-2} \xi_{i} \eta_{i}^{\beta-\alpha-1}<1, f \in C([0,1] \times[0,+\infty),[0,+\infty))$. The existence and multiplicity of positive solutions for system (1.4) were established via the monotone iterative method and the fixed point index theory.

The system of fractional differential equations boundary value problems with $p$ Laplacian operator have also received much attention and have developed very rapidly, see [24-32]. In [24], Li et al. studied the following fractional differential system involving the $p$-Laplacian operator and nonlocal boundary conditions:

$$
\left\{\begin{array}{l}
D_{0^{+}}^{\alpha_{1}}\left(\varphi_{p_{1}}\left(D_{0^{+}}^{\beta_{1}} u(t)\right)\right)=f(t, v(t)), \quad 0<t<1, \\
D_{0^{+}}^{\alpha_{2}}\left(\varphi_{p_{2}}\left(D_{0^{+}}^{\beta_{2}} v(t)\right)=g(t, u(t)), \quad 0<t<1,\right. \\
u(0)=D_{0^{+}}^{\beta_{1}} u(0)=0, \quad D_{0^{+}}^{\gamma_{1}} u(1)=\sum_{j=1}^{m-2} a_{1 j} D_{0^{+}}^{\gamma_{1}} u\left(\eta_{j}\right), \\
v(0)=D_{0^{+}}^{\beta_{2}} v(0)=0, \quad D_{0^{+}}^{\gamma_{2}} v(1)=\sum_{j=1}^{m-2} a_{2 j} D_{0^{+}}^{\gamma_{2}} \nu\left(\eta_{j}\right),
\end{array}\right.
$$

where $\alpha_{i}, \gamma_{i} \in(0,1], \beta_{i} \in(1,2], D_{0^{+}}^{\alpha_{i}}, D_{0^{+}}^{\beta_{i}}$ and $D_{0^{+}}^{\gamma_{i}}$ are the standard Riemann-Liouville derivatives, $i=1,2$. The conditions for the existence of the maximal and minimal solutions to the system were established.

Ren et al. [25] considered the following nonlocal fractional differential system:

$$
\left\{\begin{array}{l}
-D_{0^{+}}^{\alpha_{1}}\left(\varphi_{p_{1}}\left(-D_{0^{+}}^{\beta_{1}} u(t)\right)\right)=f(u(t), v(t)), \quad 0<t<1, \\
-D_{0^{+}}^{\alpha_{2}}\left(\varphi_{p_{2}}\left(-D_{0^{+}}^{\beta_{2}} v(t)\right)\right)=g(u(t), v(t)), \quad 0<t<1, \\
u(0)=D_{0^{+}}^{\beta_{1}} u(0)=D_{0^{+}}^{\beta_{1}} u(1)=0, \quad u(1)=\int_{0}^{1} u(s) d A(s), \\
v(0)=D_{0^{+}}^{\beta_{2}} v(0)=D_{0^{+}}^{\beta_{2}} v(1)=0, \quad v(1)=\int_{0}^{1} v(s) d B(s),
\end{array}\right.
$$

where $\alpha_{i}, \beta_{i} \in(1,2], D_{0^{+}}^{\alpha_{i}}$ and $D_{0^{+}}^{\beta_{i}}$ are the standard Riemann-Liouville derivatives. $A$ and $B$ are functions of bounded variations, $\int_{0}^{1} u(s) d A(s)$ and $\int_{0}^{1} v(s) d B(s)$ are Riemann-Stieltjes integrals. By introducing a new type of growth conditions and using the monotone iterative technique, some new results about the existence of maximal and minimal solutions were established, and the estimation of the lower and upper bounds of the maximum and minimum solutions was also derived.

By means of the Avery-Henderson fixed point theorem and six functionals fixed point theorem, Rao [26] investigated the existence of multiple positive solutions for a coupled system of $p$-Laplacian fractional order two point boundary value problems

$$
\left\{\begin{array}{l}
-D_{a^{+}}^{\alpha_{1}}\left(\varphi_{p}\left(D_{a^{+}}^{\beta_{1}} u(t)\right)\right)=f(t, u(t), v(t)), \quad a<t<b, \\
-D_{a^{+}}^{\alpha_{2}}\left(\varphi_{p}\left(D_{a^{+}}^{\beta_{2}} v(t)\right)\right)=g(t, u(t), v(t)), \quad a<t<b, \\
\xi u(a)-\eta u^{\prime}(a)=0, \quad \gamma u(b)+\delta u^{\prime}(b)=0, \quad D_{a^{+}}^{\beta_{1}} u(a)=0, \\
\xi v(a)-\eta v^{\prime}(a)=0, \quad \gamma v(b)+\delta v^{\prime}(b)=0, \quad D_{a^{+}}^{\beta_{2}} v(a)=0,
\end{array}\right.
$$

where $\alpha_{i} \in(0,1], \beta_{i} \in(1,2], D_{a^{+}}^{\alpha_{i}}$ and $D_{a^{+}}^{\beta_{i}}$ are the standard Riemann-Liouville derivatives. 
He and Song [29] discussed the following fractional order differential system with $p$ Laplacian operator:

$$
\left\{\begin{array}{l}
D_{0^{+}}^{\alpha_{1}}\left(\varphi_{p_{1}}\left(D_{0^{+}}^{\beta_{1}} u(t)\right)\right)=\lambda f(t, v(t)), \quad 0<t<1, \\
D_{0^{+}}^{\alpha_{2}}\left(\varphi_{p_{2}}\left(D_{0^{+}}^{\beta_{2}} v(t)\right)=\mu g(t, u(t)), \quad 0<t<1,\right. \\
u(0)=0, u(1)=a_{1} u\left(\xi_{1}\right), \quad D_{0^{+}}^{\beta_{1}} u(0)=0, \quad D_{0^{+}}^{\beta_{1}} u(1)=b_{1} D_{0^{+}}^{\beta_{1}} u\left(\eta_{1}\right), \\
v(0)=0, v(1)=a_{2} v\left(\xi_{2}\right), \quad D_{0^{+}}^{\beta_{2}} v(0)=0, \quad D_{0^{+}}^{\beta_{2}} v(1)=b_{2} D_{0^{+}}^{\beta_{2}} v\left(\eta_{2}\right),
\end{array}\right.
$$

where $\alpha_{i}, \beta_{i} \in(1,2], D_{0^{+}}^{\alpha_{i}}$ and $D_{0^{+}}^{\beta_{i}}$ are the standard Riemann-Liouville derivatives, $\xi_{i}, \eta_{i} \in$ $(0,1), a_{i}, b_{i} \in[0,1], i=1,2 . \lambda$ and $\mu$ are positive parameters. The uniqueness of solution was established by using the Banach contraction mapping principle. Khan et al. [31] considered the existence and uniqueness of solutions to a coupled system of fractional differential equations with $p$-Laplacian operator. The functions involved in the proposed coupled system were continuous and satisfied certain growth conditions. By using topological degree theory, some conditions were established which ensured the existence and uniqueness of solution to the proposed problem.

Motivated by the papers mentioned above, in this paper, we study the existence of positive solutions for a system of nonlinear fractional differential equations nonlocal boundary value problems with parameters and $p$-Laplacian operator. Under different combinations of superlinearity and sublinearity of the functions $f$ and $g$, various existence results for positive solutions are derived in terms of different values of $\lambda$ and $\mu$ via the Guo-Krasnosel'skii fixed point theorem. Moreover, in this paper it is possible to replace the four point boundary conditions by multi-point boundary conditions or integral boundary conditions with minor modifications.

\section{Preliminaries and lemmas}

We present here the definitions, some lemmas from the theory of fractional calculus and some auxiliary results that will be used to prove our main theorems.

Definition 2.1 ([1-3]) The Riemann-Liouville fractional integral of order $\alpha>0$ of a function $f:(0,+\infty) \rightarrow(-\infty,+\infty)$ is given by

$$
I_{0^{+}}^{\alpha} f(t)=\frac{1}{\Gamma(\alpha)} \int_{0}^{t}(t-s)^{\alpha-1} f(s) d s
$$

provided the right-hand side is pointwise defined on $(0,+\infty)$.

Definition $2.2([1-3])$ The Riemann-Liouville fractional derivative of order $\alpha>0$ of a continuous function $f:(0,+\infty) \rightarrow(-\infty,+\infty)$ is given by

$$
D_{0^{+}}^{\alpha} f(t)=\frac{1}{\Gamma(n-\alpha)}\left(\frac{d}{d t}\right)^{n} \int_{0}^{t}(t-s)^{n-\alpha-1} f(s) d s
$$

where $n$ is the smallest integer not less than $\alpha$, provided the right-hand side is pointwise defined on $(0,+\infty)$. 
Lemma 2.1 ([1-3]) Let $\alpha>0$. Then the following equality holds for $u \in L(0,1)$ and $D_{0^{+}}^{\alpha} u(t) \in L(0,1)$ :

$$
I_{0^{+}}^{\alpha} D_{0^{+}}^{\alpha} u(t)=u(t)+c_{1} t^{\alpha-1}+c_{2} t^{\alpha-2}+\cdots+c_{n} t^{\alpha-n}
$$

where $c_{1}, c_{2}, \ldots, c_{n} \in(-\infty,+\infty), n-1<\alpha \leq n$.

We transform problem (1.1) to its equivalent integral equations. Denote $\varphi_{p_{1}}\left(D_{0^{+}}^{\beta_{1}} u(t)\right)=$ $z(t)$, then

$$
z(0)=0, \quad z(1)=b_{1}^{p_{1}-1} z\left(\eta_{1}\right)
$$

We now consider the following fractional differential equation:

$$
\left\{\begin{array}{l}
-D_{0^{+}}^{\alpha_{1}} z(t)=y(t), \quad 0<t<1 \\
z(0)=0, \quad z(1)=b_{1}^{p_{1}-1} z\left(\eta_{1}\right) .
\end{array}\right.
$$

Lemma 2.2 ([17]) If $y \in C[0,1]$, then problem (2.1) has a unique solution

$$
z(t)=\int_{0}^{1} H_{1}(t, s) y(s) d s
$$

where

$$
\begin{aligned}
& H_{1}(t, s)=h_{1}(t, s)+\frac{b_{1}^{p_{1}-1} t^{\alpha_{1}-1}}{1-b_{1}^{p_{1}-1} \eta_{1}^{\alpha_{1}-1}} h_{1}\left(\eta_{1}, s\right), \\
& h_{1}(t, s)=\frac{1}{\Gamma\left(\alpha_{1}\right)} \begin{cases}{[t(1-s)]^{\alpha_{1}-1},} & 0 \leq t \leq s \leq 1, \\
{[t(1-s)]^{\alpha_{1}-1}-(t-s)^{\alpha_{1}-1},} & 0 \leq s \leq t \leq 1 .\end{cases}
\end{aligned}
$$

From the above analysis, the boundary value problem

$$
\left\{\begin{array}{l}
-D_{0^{+}}^{\alpha_{1}}\left(\varphi_{p_{1}}\left(D_{0^{+}}^{\beta_{1}} u(t)\right)\right)=y(t), \quad 0<t<1 \\
u(0)=u(1)=u^{\prime}(0)=u^{\prime}(1)=0, \quad D_{0^{+}}^{\beta_{1}} u(0)=0, \quad D_{0^{+}}^{\beta_{1}} u(1)=b_{1} D_{0^{+}}^{\beta_{1}} u\left(\eta_{1}\right)
\end{array}\right.
$$

is equal to

$$
\left\{\begin{array}{l}
D_{0^{+}}^{\beta_{1}} u(t)=\varphi_{q_{1}}\left(\int_{0}^{1} H_{1}(t, s) y(s) d s\right) \\
u(0)=u(1)=u^{\prime}(0)=u^{\prime}(1)=0
\end{array}\right.
$$

Lemma 2.3 ([17]) If $y \in C[0,1]$, then problem (2.2) has a unique solution

$$
u(t)=\int_{0}^{1} G_{1}(t, s) \varphi_{q_{1}}\left(\int_{0}^{1} H_{1}(s, \tau) y(\tau) d \tau\right) d s
$$


where

$$
G_{1}(t, s)=\frac{1}{\Gamma\left(\beta_{1}\right)}\left\{\begin{array}{c}
t^{\beta_{1}-2}(1-s)^{\beta_{1}-2}\left[(s-t)+\left(\beta_{1}-2\right)(1-t) s\right] \\
0 \leq t \leq s \leq 1 \\
t^{\beta_{1}-2}(1-s)^{\beta_{1}-2}\left[(s-t)+\left(\beta_{1}-2\right)(1-t) s\right]+(t-s)^{\beta_{1}-1} \\
0 \leq s \leq t \leq 1
\end{array}\right.
$$

Lemma $2.4([17,33])$ The function $G_{1}(t, s)$ defined by $(2.3)$ is continuous on $[0,1] \times[0,1]$ and has the following properties:

(a) $G_{1}(t, s)>0$ for all $(t, s) \in(0,1) \times(0,1)$;

(b) $\left(\beta_{1}-2\right) k_{1}(t) l_{1}(s) \leq \Gamma\left(\beta_{1}\right) G_{1}(t, s) \leq M_{1} l_{1}(s),(t, s) \in(0,1) \times(0,1)$;

(c) $\left(\beta_{1}-2\right) k_{1}(t) l_{1}(s) \leq \Gamma\left(\beta_{1}\right) G_{1}(t, s) \leq M_{1} k_{1}(t),(t, s) \in(0,1) \times(0,1)$; where

$$
\begin{aligned}
& k_{1}(t)=t^{\beta_{1}-2}(1-t)^{2}, \quad l_{1}(s)=s^{2}(1-s)^{\beta_{1}-2}, \\
& M_{1}=\max \left\{\beta_{1}-1,\left(\beta_{1}-2\right)^{2}\right\} .
\end{aligned}
$$

Similarly, we can obtain the following Lemmas 2.5 and 2.6 for the following boundary value problem:

$$
\left\{\begin{array}{l}
-D_{0^{+}}^{\alpha_{2}}\left(\varphi_{p_{2}}\left(D_{0^{+}}^{\beta_{2}} v(t)\right)\right)=y(t), \quad 0<t<1, \\
v(0)=v(1)=v^{\prime}(0)=v^{\prime}(1)=0, \quad D_{0^{+}}^{\beta_{2}} \nu(0)=0, \quad D_{0^{+}}^{\beta_{2}} v(1)=b_{2} D_{0^{+}}^{\beta_{2}} u\left(\eta_{2}\right) .
\end{array}\right.
$$

Lemma 2.5 If $y \in C[0,1]$, then problem (2.4) has a unique solution

$$
\nu(t)=\int_{0}^{1} G_{2}(t, s) \varphi_{q_{2}}\left(\int_{0}^{1} H_{2}(s, \tau) y(\tau) d \tau\right) d s,
$$

where

$$
\begin{aligned}
& G_{2}(t, s)=\frac{1}{\Gamma\left(\beta_{2}\right)}\left\{\begin{array}{l}
t^{\beta_{2}-2}(1-s)^{\beta_{2}-2}\left[(s-t)+\left(\beta_{2}-2\right)(1-t) s\right] \\
0 \leq t \leq s \leq 1, \\
t^{\beta_{2}-2}(1-s)^{\beta_{2}-2}\left[(s-t)+\left(\beta_{2}-2\right)(1-t) s\right]+(t-s)^{\beta_{2}-1} \\
0 \leq s \leq t \leq 1,
\end{array}\right. \\
& H_{2}(t, s)=h_{2}(t, s)+\frac{b_{2}^{p_{2}-1} t^{\alpha_{2}-1}}{1-b_{2}^{p_{2}-1} \eta_{2}^{\alpha_{2}-1}} h_{1}\left(\eta_{2}, s\right), \\
& h_{2}(t, s)=\frac{1}{\Gamma\left(\alpha_{2}\right)} \begin{cases}{[t(1-s)]^{\alpha_{2}-1},} & 0 \leq t \leq s \leq 1, \\
{[t(1-s)]^{\alpha_{2}-1}-(t-s)^{\alpha_{2}-1},} & 0 \leq s \leq t \leq 1 .\end{cases}
\end{aligned}
$$

Lemma 2.6 The function $G_{2}(t, s)$ given by $(2.5)$ has the properties:

(a) $G_{2}(t, s)>0,(t, s) \in(0,1) \times(0,1)$;

(b) $\left(\beta_{2}-2\right) k_{2}(t) l_{2}(s) \leq \Gamma\left(\beta_{2}\right) G_{2}(t, s) \leq M_{2} l_{2}(s),(t, s) \in(0,1) \times(0,1)$;

(c) $\left(\beta_{2}-2\right) k_{2}(t) l_{2}(s) \leq \Gamma\left(\beta_{2}\right) G_{2}(t, s) \leq M_{2} k_{2}(t),(t, s) \in(0,1) \times(0,1)$; 
where

$$
k_{2}(t)=t^{\beta_{2}-2}(1-t)^{2}, \quad l_{2}(s)=s^{2}(1-s)^{\beta_{2}-2}, \quad M_{2}=\max \left\{\beta_{2}-1,\left(\beta_{2}-2\right)^{2}\right\} .
$$

Let $X=C[0,1]$, then $X$ is a Banach space with the norm $\|u\|=\sup _{t \in[0,1]}|u(t)|$. Let $Y=$ $X \times X$, then $Y$ is a Banach space with the norm $\|(u, v)\|_{Y}=\|u\|+\|v\|$. For $\theta_{1}, \theta_{2} \in(0,1)$ and $\theta_{1}<\theta_{2}$, denote

$$
P=\left\{(u, v) \in Y: u(t) \geq 0, v(t) \geq 0, \forall t \in[0,1], \min _{t \in\left[\theta_{1}, \theta_{2}\right]}(u(t)+v(t)) \geq \gamma\|(u, v)\|_{Y}\right\},
$$

where $\gamma=\min \left\{\gamma_{1}, \gamma_{2}\right\}$ and $\gamma_{i}=\frac{\left(\beta_{i}-2\right)}{M_{i}} \min _{t \in\left[\theta_{1}, \theta_{2}\right]} k_{i}(t), i=1,2$, then $P$ is a cone of $Y$. Define operators $T_{1}, T_{2}: Y \rightarrow X$ and $Q: Y \rightarrow Y$ as follows:

$$
\begin{aligned}
& T_{1}(u, v)(t)=\varphi_{q_{1}}(\lambda) \int_{0}^{1} G_{1}(t, s) \varphi_{q_{1}}\left(\int_{0}^{1} H_{1}(s, \tau) f(\tau, u(\tau), v(\tau)) d \tau\right) d s, \quad t \in[0,1], \\
& T_{2}(u, v)(t)=\varphi_{q_{2}}(\mu) \int_{0}^{1} G_{2}(t, s) \varphi_{q_{2}}\left(\int_{0}^{1} H_{2}(s, \tau) g(\tau, u(\tau), v(\tau)) d \tau\right) d s, \quad t \in[0,1], \\
& Q(u, v)=\left(T_{1}(u, v), T_{2}(u, v)\right), \quad(u, v) \in Y .
\end{aligned}
$$

It is well known that if $(u, v)$ is a fixed point of the operator $Q$ in $P$, then $(u, v)$ is a positive solution of system (1.1).

Lemma 2.7 $Q: P \rightarrow P$ is a completely continuous operator.

Proof For $(u, v) \in P$ and $t \in[0,1]$, obviously, $T_{1}(u, v)(t) \geq 0, T_{2}(u, v)(t) \geq 0$. It follows from Lemmas 2.4 and 2.6 that

$$
\begin{aligned}
T_{1}(u, v)(t) & =\varphi_{q_{1}}(\lambda) \int_{0}^{1} G_{1}(t, s) \varphi_{q_{1}}\left(\int_{0}^{1} H_{1}(s, \tau) f(\tau, u(\tau), v(\tau)) d \tau\right) d s \\
& \leq \varphi_{q_{1}}(\lambda) \int_{0}^{1} \frac{M_{1} l_{1}(s)}{\Gamma\left(\beta_{1}\right)} \varphi_{q_{1}}\left(\int_{0}^{1} H_{1}(s, \tau) f(\tau, u(\tau), v(\tau)) d \tau\right) d s
\end{aligned}
$$

then

$$
\left\|T_{1}(u, v)\right\| \leq \frac{M_{1}}{\Gamma\left(\beta_{1}\right)} \varphi_{q_{1}}(\lambda) \int_{0}^{1} l_{1}(s) \varphi_{q_{1}}\left(\int_{0}^{1} H_{1}(s, \tau) f(\tau, u(\tau), v(\tau)) d \tau\right) d s .
$$

Similarly,

$$
\left\|T_{2}(u, v)\right\| \leq \frac{M_{2}}{\Gamma\left(\beta_{2}\right)} \varphi_{q_{2}}(\mu) \int_{0}^{1} l_{2}(s) \varphi_{q_{2}}\left(\int_{0}^{1} H_{2}(s, \tau) g(\tau, u(\tau), v(\tau)) d \tau\right) d s .
$$

Therefore

$$
\begin{aligned}
\|Q(u, v)\|_{Y} \leq & \frac{M_{1}}{\Gamma\left(\beta_{1}\right)} \varphi_{q_{1}}(\lambda) \int_{0}^{1} l_{1}(s) \varphi_{q_{1}}\left(\int_{0}^{1} H_{1}(s, \tau) f(\tau, u(\tau), v(\tau)) d \tau\right) d s \\
& +\frac{M_{2}}{\Gamma\left(\beta_{2}\right)} \varphi_{q_{2}}(\mu) \int_{0}^{1} l_{2}(s) \varphi_{q_{2}}\left(\int_{0}^{1} H_{2}(s, \tau) g(\tau, u(\tau), v(\tau)) d \tau\right) d s .
\end{aligned}
$$


On the other hand, for any $t \in\left[\theta_{1}, \theta_{2}\right]$, by Lemma 2.4, we have

$$
\begin{aligned}
T_{1}(u, v)(t) & \geq \varphi_{q_{1}}(\lambda) \int_{0}^{1} \frac{\beta_{1}-2}{\Gamma\left(\beta_{1}\right)} k_{1}(t) l_{1}(s) \varphi_{q_{1}}\left(\int_{0}^{1} H_{1}(s, \tau) f(\tau, u(\tau), v(\tau)) d \tau\right) d s \\
& =\frac{\beta_{1}-2}{\Gamma\left(\beta_{1}\right)} k_{1}(t) \varphi_{q_{1}}(\lambda) \int_{0}^{1} l_{1}(s) \varphi_{q_{1}}\left(\int_{0}^{1} H_{1}(s, \tau) f(\tau, u(\tau), v(\tau)) d \tau\right) d s \\
& \geq \frac{\beta_{1}-2}{M_{1}} k_{1}(t)\left\|T_{1}(u, v)\right\|
\end{aligned}
$$

then

$$
\min _{t \in\left[\theta_{1}, \theta_{2}\right]} T_{1}(u, v)(t) \geq \gamma_{1}\left\|T_{1}(u, v)\right\|
$$

Similarly,

$$
\min _{t \in\left[\theta_{1}, \theta_{2}\right]} T_{2}(u, v)(t) \geq \gamma_{2}\left\|T_{2}(u, v)\right\|
$$

Hence

$$
\begin{aligned}
& \min _{t \in\left[\theta_{1}, \theta_{2}\right]}\left(T_{1}(u, v)(t)+T_{2}(u, v)(t)\right) \\
& \geq \min _{t \in\left[\theta_{1}, \theta_{2}\right]} T_{1}(u, v)(t)+\min _{t \in\left[\theta_{1}, \theta_{2}\right]} T_{2}(u, v)(t) \\
& \geq \gamma_{1}\left\|T_{1}(u, v)\right\|+\gamma_{2}\left\|T_{2}(u, v)\right\| \\
& \geq \gamma\left(\left\|T_{1}(u, v)\right\|+\left\|T_{2}(u, v)\right\|\right)=\gamma\|Q(u, v)\|_{Y},
\end{aligned}
$$

that is, $Q(P) \subset P$.

By the Ascoli-Arzela theorem and the continuity of $f, g, G_{i}$ and $H_{i}$, we deduce that $T_{1}$ and $T_{2}$ are completely continuous operators, then $Q$ is a completely continuous operator. This completes the proof.

Lemma 2.8 ([34]) Let $P$ be a positive cone in a Banach space $E, \Omega_{1}$ and $\Omega_{2}$ are bounded open sets in $E, \theta \in \Omega_{1}, \bar{\Omega}_{1} \subset \Omega_{2}, A: P \cap\left(\bar{\Omega}_{2} \backslash \Omega_{1}\right) \rightarrow P$ is a completely continuous operator. If the following conditions are satisfied:

(i) $\|A x\| \leq\|x\|, \forall x \in P \cap \partial \Omega_{1},\|A x\| \geq\|x\|, \forall x \in P \cap \partial \Omega_{2}$, or

(ii) $\|A x\| \geq\|x\|, \forall x \in P \cap \partial \Omega_{1},\|A x\| \leq\|x\|, \forall x \in P \cap \partial \Omega_{2}$, then $A$ has at least one fixed point in $P \cap\left(\bar{\Omega}_{2} \backslash \Omega_{1}\right)$.

\section{Main results}

Denote

$$
\begin{array}{ll}
f_{0}^{s}=\limsup _{u+v \rightarrow 0} \max _{t \in[0,1]} \frac{f(t, u, v)}{\varphi_{p_{1}}(u+v)}, & g_{0}^{s}=\limsup _{u+v \rightarrow 0} \max _{t \in[0,1]} \frac{g(t, u, v)}{\varphi_{p_{2}}(u+v)}, \\
f_{0}^{i}=\liminf _{u+v \rightarrow 0} \min _{t \in\left[\theta_{1}, \theta_{2}\right]} \frac{f(t, u, v)}{\varphi_{p_{1}}(u+v)}, & g_{0}^{i}=\liminf _{u+v \rightarrow 0} \min _{t \in\left[\theta_{1}, \theta_{2}\right]} \frac{g(t, u, v)}{\varphi_{p_{2}}(u+v)}, \\
f_{\infty}^{s}=\limsup _{u+v \rightarrow \infty} \max _{t \in[0,1]} \frac{f(t, u, v)}{\varphi_{p_{1}}(u+v)}, & g_{\infty}^{s}=\limsup _{u+v \rightarrow \infty} \max _{t \in[0,1]} \frac{g(t, u, v)}{\varphi_{p_{2}}(u+v)},
\end{array}
$$




$$
\begin{aligned}
f_{\infty}^{i} & =\liminf _{u+v \rightarrow \infty} \min _{t \in\left[\theta_{1}, \theta_{2}\right]} \frac{f(t, u, v)}{\varphi_{p_{1}}(u+v)}, \quad g_{\infty}^{i}=\liminf _{u+\nu \rightarrow \infty} \min _{t \in\left[\theta_{1}, \theta_{2}\right]} \frac{g(t, u, v)}{\varphi_{p_{2}}(u+v)} . \\
A & =\int_{0}^{1} l_{1}(s) \varphi_{q_{1}}\left(\int_{0}^{1} H_{1}(s, \tau) d \tau\right) d s, \quad B=\int_{0}^{1} l_{2}(s) \varphi_{q_{2}}\left(\int_{0}^{1} H_{2}(s, \tau) d \tau\right) d s, \\
C & =\int_{\theta_{1}}^{\theta_{2}} l_{1}(s) \varphi_{q_{1}}\left(\int_{\theta_{1}}^{\theta_{2}} H_{1}(s, \tau) d \tau\right) d s, \quad D=\int_{\theta_{1}}^{\theta_{2}} l_{2}(s) \varphi_{q_{2}}\left(\int_{\theta_{1}}^{\theta_{2}} H_{2}(s, \tau) d \tau\right) d s .
\end{aligned}
$$

For $f_{0}^{s}, g_{0}^{s}, f_{\infty}^{i}, g_{\infty}^{i} \in(0, \infty)$, we define the symbols $L_{1}, L_{2}, L_{3}$ and $L_{4}$ as follows:

$$
\begin{array}{ll}
L_{1}=\varphi_{p_{1}}\left(\frac{\Gamma\left(\beta_{1}\right)}{2\left(\beta_{1}-2\right) C \gamma_{1} \gamma}\right) \frac{1}{f_{\infty}^{i}}, & L_{2}=\varphi_{p_{1}}\left(\frac{\Gamma\left(\beta_{1}\right)}{2 A M_{1}}\right) \frac{1}{f_{0}^{s}}, \\
L_{3}=\varphi_{p_{2}}\left(\frac{\Gamma\left(\beta_{2}\right)}{2\left(\beta_{2}-2\right) D \gamma_{2} \gamma}\right) \frac{1}{g_{\infty}^{i}}, & L_{4}=\varphi_{p_{2}}\left(\frac{\Gamma\left(\beta_{2}\right)}{2 B M_{2}}\right) \frac{1}{g_{0}^{s}} .
\end{array}
$$

Theorem 3.1 (1) If $f_{0}^{s}, g_{0}^{s}, f_{\infty}^{i}, g_{\infty}^{i} \in(0, \infty), L_{1}<L_{2}, L_{3}<L_{4}$, then for each $\lambda \in\left(L_{1}, L_{2}\right)$ and $\mu \in\left(L_{3}, L_{4}\right)$, system (1.1) has at least one positive solution $(u(t), v(t)), t \in(0,1)$.

(2) If $f_{0}^{s}=0, g_{0}^{s}, f_{\infty}^{i}, g_{\infty}^{i} \in(0, \infty), L_{3}<L_{4}$, then for each $\lambda \in\left(L_{1}, \infty\right)$ and $\mu \in\left(L_{3}, L_{4}\right)$, system (1.1) has at least one positive solution $(u(t), v(t)), t \in(0,1)$.

(3) If $g_{0}^{s}=0, f_{0}^{s}, f_{\infty}^{i}, g_{\infty}^{i} \in(0, \infty), L_{1}<L_{2}$, then for each $\lambda \in\left(L_{1}, L_{2}\right)$ and $\mu \in\left(L_{3}, \infty\right)$, system (1.1) has at least one positive solution $(u(t), v(t)), t \in(0,1)$.

(4) If $f_{0}^{s}=g_{0}^{s}=0, f_{\infty}^{i}, g_{\infty}^{i} \in(0, \infty)$, then for each $\lambda \in\left(L_{1}, \infty\right)$ and $\mu \in\left(L_{3}, \infty\right)$, system (1.1) has at least one positive solution $(u(t), v(t)), t \in(0,1)$.

(5) If $\left\{f_{0}^{s}, g_{0}^{s} \in(0, \infty), f_{\infty}^{i}=\infty\right\}$ or $\left\{f_{0}^{s}, g_{0}^{s} \in(0, \infty), g_{\infty}^{i}=\infty\right\}$, then for each $\lambda \in\left(0, L_{2}\right)$ and $\mu \in\left(0, L_{4}\right)$, system $(1.1)$ has at least one positive solution $(u(t), v(t)), t \in(0,1)$.

(6) If $\left\{f_{0}^{s}=0, g_{0}^{s} \in(0, \infty), g_{\infty}^{i}=\infty\right\}$ or $\left\{f_{0}^{s}=0, g_{0}^{s} \in(0, \infty), f_{\infty}^{i}=\infty\right\}$, then for each $\lambda \in(0, \infty)$ and $\mu \in\left(0, L_{4}\right)$, system (1.1) has at least one positive solution $(u(t), v(t)), t \in(0,1)$.

(7) If $\left\{f_{0}^{s} \in(0, \infty), g_{0}^{s}=0, g_{\infty}^{i}=\infty\right\}$ or $\left\{f_{0}^{s} \in(0, \infty), g_{0}^{s}=0, f_{\infty}^{i}=\infty\right\}$, then for each $\lambda \in\left(0, L_{2}\right)$ and $\mu \in(0, \infty)$, system (1.1) has at least one positive solution $(u(t), v(t)), t \in(0,1)$.

(8) If $\left\{f_{0}^{s}=g_{0}^{s}=0, g_{\infty}^{i}=\infty\right\}$ or $\left\{f_{0}^{s}=g_{0}^{s}=0, f_{\infty}^{i}=\infty\right\}$, then for each $\lambda \in(0, \infty)$ and $\mu \in$ $(0, \infty)$, system (1.1) has at least one positive solution $(u(t), v(t)), t \in(0,1)$.

Proof Because the proofs of the above cases are similar, in what follows we will prove two of them, namely cases (1) and (6).

(1) For any $\lambda \in\left(L_{1}, L_{2}\right)$ and $\mu \in\left(L_{3}, L_{4}\right)$, there exists $0<\varepsilon<\min \left\{f_{\infty}^{i}, g_{\infty}^{i}\right\}$ such that

$$
\begin{aligned}
& \varphi_{p_{1}}\left(\frac{\Gamma\left(\beta_{1}\right)}{2\left(\beta_{1}-2\right) C \gamma_{1} \gamma}\right) \frac{1}{f_{\infty}^{i}-\varepsilon} \leq \lambda \leq \varphi_{p_{1}}\left(\frac{\Gamma\left(\beta_{1}\right)}{2 A M_{1}}\right) \frac{1}{f_{0}^{s}+\varepsilon}, \\
& \varphi_{p_{2}}\left(\frac{\Gamma\left(\beta_{2}\right)}{2\left(\beta_{2}-2\right) D \gamma_{2} \gamma}\right) \frac{1}{g_{\infty}^{i}-\varepsilon} \leq \mu \leq \varphi_{p_{2}}\left(\frac{\Gamma\left(\beta_{2}\right)}{2 B M_{2}}\right) \frac{1}{g_{0}^{s}+\varepsilon} .
\end{aligned}
$$

By the definitions of $f_{0}^{s}$ and $g_{0}^{s}$, there exists $R_{1}>0$ such that

$$
\begin{array}{ll}
f(t, u, v)<\left(f_{0}^{s}+\varepsilon\right) \varphi_{p_{1}}(u+v), & t \in[0,1], 0 \leq u+v \leq R_{1}, \\
g(t, u, v)<\left(g_{0}^{s}+\varepsilon\right) \varphi_{p_{2}}(u+v), & t \in[0,1], 0 \leq u+v \leq R_{1} .
\end{array}
$$


Denote $\Omega_{1}=\left\{(u, v) \in Y:\|(u, v)\|_{Y}<R_{1}\right\}$, for any $(u, v) \in P \cap \partial \Omega_{1}$ and $t \in[0,1]$, we have $0 \leq u(t)+v(t) \leq\|u\|+\|v\|=\|(u, v)\|_{Y}=R_{1}$, then

$$
\begin{aligned}
T_{1}(u, v)(t) & =\varphi_{q_{1}}(\lambda) \int_{0}^{1} G_{1}(t, s) \varphi_{q_{1}}\left(\int_{0}^{1} H_{1}(s, \tau) f(\tau, u(\tau), v(\tau)) d \tau\right) d s \\
& \leq \varphi_{q_{1}}(\lambda) \int_{0}^{1} \frac{M_{1}}{\Gamma\left(\beta_{1}\right)} l_{1}(s) \varphi_{q_{1}}\left(\int_{0}^{1} H_{1}(s, \tau)\left(f_{0}^{s}+\varepsilon\right) \varphi_{p_{1}}(u(\tau)+v(\tau)) d \tau\right) d s \\
& \leq \frac{M_{1}}{\Gamma\left(\beta_{1}\right)} \varphi_{q_{1}}(\lambda) \varphi_{q_{1}}\left(f_{0}^{s}+\varepsilon\right) \int_{0}^{1} l_{1}(s) \varphi_{q_{1}}\left(\int_{0}^{1} H_{1}(s, \tau) \varphi_{p_{1}}(\|u\|+\|v\|) d \tau\right) d s \\
& =\frac{M_{1}}{\Gamma\left(\beta_{1}\right)} \varphi_{q_{1}}\left(\lambda\left(f_{0}^{s}+\varepsilon\right)\right) A\|(u, v)\|_{Y} \\
& \leq \frac{1}{2}\|(u, v)\|_{Y},
\end{aligned}
$$

so $\left\|T_{1}(u, v)\right\| \leq \frac{1}{2}\|(u, v)\|_{Y},(u, v) \in P \cap \partial \Omega_{1}$. In a similar manner, we deduce

$$
\begin{aligned}
T_{2}(u, v)(t) & =\varphi_{q_{2}}(\mu) \int_{0}^{1} G_{2}(t, s) \varphi_{q_{2}}\left(\int_{0}^{1} H_{2}(s, \tau) g(\tau, u(\tau), v(\tau)) d \tau\right) d s \\
& \leq \varphi_{q_{2}}(\mu) \int_{0}^{1} \frac{M_{2}}{\Gamma\left(\beta_{2}\right)} l_{2}(s) \varphi_{q_{2}}\left(\int_{0}^{1} H_{2}(s, \tau)\left(g_{0}^{s}+\varepsilon\right) \varphi_{p_{2}}(u(\tau)+v(\tau)) d \tau\right) d s \\
& \leq \frac{M_{2}}{\Gamma\left(\beta_{2}\right)} \varphi_{q_{2}}\left(\mu\left(g_{0}^{s}+\varepsilon\right)\right) \int_{0}^{1} l_{2}(s) \varphi_{q_{2}}\left(\int_{0}^{1} H_{2}(s, \tau) d \tau\right) d s\|(u, v)\|_{Y} \\
& =\frac{M_{2}}{\Gamma\left(\beta_{2}\right)} \varphi_{q_{2}}\left(\mu\left(g_{0}^{s}+\varepsilon\right)\right) B\|(u, v)\|_{Y} \leq \frac{1}{2}\|(u, v)\|_{Y},
\end{aligned}
$$

then $\left\|T_{2}(u, v)\right\| \leq \frac{1}{2}\|(u, v)\|_{Y},(u, v) \in P \cap \partial \Omega_{1}$. Hence

$$
\|Q(u, v)\|_{Y}=\left\|T_{1}(u, v)\right\|+\left\|T_{2}(u, v)\right\| \leq\|(u, v)\|_{Y}, \quad(u, v) \in P \cap \partial \Omega_{1} .
$$

On the other hand, by the definitions of $f_{\infty}^{i}$ and $g_{\infty}^{i}$, there exists $\overline{R_{2}}>0$ such that

$$
\begin{aligned}
& f(t, u, v) \geq\left(f_{\infty}^{i}-\varepsilon\right) \varphi_{p_{1}}(u+v), \quad t \in\left[\theta_{1}, \theta_{2}\right], u, v \geq 0, u+v \geq \overline{R_{2}}, \\
& g(t, u, v) \geq\left(g_{\infty}^{i}-\varepsilon\right) \varphi_{p_{2}}(u+v), \quad t \in\left[\theta_{1}, \theta_{2}\right], u, v \geq 0, u+v \geq \overline{R_{2}} .
\end{aligned}
$$

Denote $R_{2}=\max \left\{2 R_{1}, \overline{R_{2}}\right\}$ and $\Omega_{2}=\left\{(u, v) \in Y:\|(u, v)\|_{Y}<R_{2}\right\}$. For any $(u, v) \in P \cap \partial \Omega_{2}$, we have $\min _{t \in\left[\theta_{1}, \theta_{2}\right]}(u(t)+v(t)) \geq \gamma\|(u, v)\|_{Y}=\gamma R_{2} \geq \overline{R_{2}}$, then

$$
\begin{aligned}
& T_{1}(u, v)\left(\theta_{1}\right) \\
& \quad=\varphi_{q_{1}}(\lambda) \int_{0}^{1} G_{1}\left(\theta_{1}, s\right) \varphi_{q_{1}}\left(\int_{0}^{1} H_{1}(s, \tau) f(\tau, u(\tau), v(\tau)) d \tau\right) d s \\
& \quad \geq \varphi_{q_{1}}(\lambda) \int_{0}^{1} \frac{\beta_{1}-2}{\Gamma\left(\beta_{1}\right)} \gamma_{1} l_{1}(s) \varphi_{q_{1}}\left(\int_{\theta_{1}}^{\theta_{2}} H_{1}(s, \tau) f(\tau, u(\tau), v(\tau)) d \tau\right) d s \\
& \quad \geq \frac{\beta_{1}-2}{\Gamma\left(\beta_{1}\right)} \gamma_{1} \varphi_{q_{1}}(\lambda) \int_{\theta_{1}}^{\theta_{2}} l_{1}(s) \varphi_{q_{1}}\left(\int_{\theta_{1}}^{\theta_{2}} H_{1}(s, \tau)\left(f_{\infty}^{i}-\varepsilon\right) \varphi_{p_{1}}(u(\tau)+v(\tau)) d \tau\right) d s
\end{aligned}
$$




$$
\begin{aligned}
& \geq \frac{\beta_{1}-2}{\Gamma\left(\beta_{1}\right)} \gamma_{1} \varphi_{q_{1}}\left(\lambda\left(f_{\infty}^{i}-\varepsilon\right)\right) \int_{\theta_{1}}^{\theta_{2}} l_{1}(s) \varphi_{q_{1}}\left(\int_{\theta_{1}}^{\theta_{2}} H_{1}(s, \tau) \varphi_{p_{1}}\left(\gamma\|(u, v)\|_{Y}\right) d \tau\right) d s \\
& =\frac{\beta_{1}-2}{\Gamma\left(\beta_{1}\right)} \gamma_{1} \gamma \varphi_{q_{1}}\left(\lambda\left(f_{\infty}^{i}-\varepsilon\right)\right) C\|(u, v)\|_{Y} \geq \frac{1}{2}\|(u, v)\|_{Y}
\end{aligned}
$$

and $\left\|T_{1}(u, v)\right\| \geq \frac{1}{2}\|(u, v)\|_{Y},(u, v) \in P \cap \partial \Omega_{2}$. Similarly, we have

$$
\begin{aligned}
T_{2}(u, v)\left(\theta_{2}\right) \\
\quad=\varphi_{q_{2}}(\mu) \int_{0}^{1} G_{2}\left(\theta_{2}, s\right) \varphi_{q_{2}}\left(\int_{0}^{1} H_{2}(s, \tau) g(\tau, u(\tau), v(\tau)) d \tau\right) d s \\
\quad \geq \varphi_{q_{2}}(\mu) \int_{0}^{1} \frac{\beta_{2}-2}{\Gamma\left(\beta_{2}\right)} \gamma_{2} l_{2}(s) \varphi_{q_{2}}\left(\int_{\theta_{1}}^{\theta_{2}} H_{2}(s, \tau) g(\tau, u(\tau), v(\tau)) d \tau\right) d s \\
\geq \frac{\beta_{2}-2}{\Gamma\left(\beta_{2}\right)} \gamma_{2} \varphi_{q_{2}}(\mu) \int_{0}^{1} l_{2}(s) \varphi_{q_{2}}\left(\int_{\theta_{1}}^{\theta_{2}} H_{2}(s, \tau)\left(g_{\infty}^{i}-\varepsilon\right) \varphi_{p_{2}}(u(\tau)+v(\tau)) d \tau\right) d s \\
\geq \frac{\beta_{2}-2}{\Gamma\left(\beta_{2}\right)} \gamma_{2} \varphi_{q_{2}}(\mu) \int_{\theta_{1}}^{\theta_{2}} l_{2}(s) \varphi_{q_{2}}\left(\int_{\theta_{1}}^{\theta_{2}} H_{2}(s, \tau)\left(g_{\infty}^{i}-\varepsilon\right) \varphi_{p_{2}}\left(\gamma\|(u, v)\|_{Y}\right) d \tau\right) d s \\
=\frac{\beta_{2}-2}{\Gamma\left(\beta_{2}\right)} \gamma_{2} \gamma \varphi_{q_{2}}\left(\mu\left(g_{\infty}^{i}-\varepsilon\right)\right) D\|(u, v)\|_{Y} \geq \frac{1}{2}\|(u, v)\|_{Y},
\end{aligned}
$$

then $\left\|T_{2}(u, v)\right\| \geq \frac{1}{2}\|(u, v)\|_{Y},(u, v) \in P \cap \partial \Omega_{2}$. So,

$$
\|Q(u, v)\|_{Y} \geq\|(u, v)\|_{Y}, \quad(u, v) \in P \cap \partial \Omega_{2} .
$$

Therefore, by (3.2), (3.3) and Lemma 2.8, we conclude that $Q$ has at least one fixed point $(u, v) \in P \cap\left(\overline{\Omega_{2}} \backslash \Omega_{1}\right)$ with $R_{1} \leq\|(u, v)\|_{Y} \leq R_{2}$.

(6) Suppose $f_{0}^{s}=0, g_{0}^{s} \in(0, \infty), g_{\infty}^{i}=\infty$, then for any $\lambda \in(0, \infty)$ and $\mu \in\left(0, L_{4}\right)$, there exists $\varepsilon>0$ such that

$$
0<\lambda<\varphi_{p_{1}}\left(\frac{\Gamma\left(\beta_{1}\right)}{2 A M_{1}}\right) \frac{1}{\varepsilon}, \quad \varphi_{p_{2}}\left(\frac{\Gamma\left(\beta_{2}\right)}{\left(\beta_{2}-2\right) D \gamma_{2} \gamma}\right) \varepsilon<\mu<\varphi_{p_{2}}\left(\frac{\Gamma\left(\beta_{2}\right)}{2 B M_{2}}\right) \frac{1}{g_{0}^{s}+\varepsilon} .
$$

By the definitions of $f_{0}^{s}$ and $g_{0}^{s}$, there exists $R_{3}>0$ such that

$$
\begin{aligned}
& f(t, u, v)<\varepsilon \varphi_{p_{1}}(u+v), \quad t \in[0,1], 0 \leq u+v \leq R_{3}, \\
& g(t, u, v)<\left(g_{0}^{s}+\varepsilon\right) \varphi_{p_{2}}(u+v), \quad t \in[0,1], 0 \leq u+v \leq R_{3} .
\end{aligned}
$$

Denote $\Omega_{3}=\left\{(u, v) \in Y:\|(u, v)\|_{Y}<R_{3}\right\}$. For any $(u, v) \in P \cap \partial \Omega_{3}$ and $t \in[0,1]$, we have

$$
\begin{aligned}
T_{1}(u, v)(t) & \leq \varphi_{q_{1}}(\lambda) \int_{0}^{1} \frac{M_{1}}{\Gamma\left(\beta_{1}\right)} l_{1}(s) \varphi_{q_{1}}\left(\int_{0}^{1} H_{1}(s, \tau) \varepsilon \varphi_{p_{1}}(u(\tau)+v(\tau)) d \tau\right) d s \\
& \leq \frac{M_{1}}{\Gamma\left(\beta_{1}\right)} \varphi_{q_{1}}(\lambda \varepsilon) \int_{0}^{1} l_{1}(s) \varphi_{q_{1}}\left(\int_{0}^{1} H_{1}(s, \tau) d \tau\right) d s\|(u, v)\|_{Y} \\
& =\frac{M_{1}}{\Gamma\left(\beta_{1}\right)} \varphi_{q_{1}}(\lambda \varepsilon) A\|(u, v)\|_{Y}<\frac{1}{2}\|(u, v)\|_{Y},
\end{aligned}
$$

then $\left\|T_{1}(u, v)\right\| \leq \frac{1}{2}\|(u, v)\|_{Y},(u, v) \in P \cap \partial \Omega_{3}$. 
Similar to (3.1) of (1), we get $\left\|T_{2}(u, v)\right\| \leq \frac{1}{2}\|(u, v)\|_{Y},(u, v) \in P \cap \partial \Omega_{3}$, then

$$
\|Q(u, v)\|_{Y} \leq\|(u, v)\|_{Y}, \quad(u, v) \in P \cap \partial \Omega_{3}
$$

On the other hand, by $g_{\infty}^{i}=\infty$, there exists $\overline{R_{4}}>0$ such that

$$
g(t, u, v) \geq \frac{1}{\varepsilon} \varphi_{p_{2}}(u+v), \quad t \in\left[\theta_{1}, \theta_{2}\right], u, v \geq 0, u+v \geq \overline{R_{4}}
$$

Let $R_{4}=\max \left\{2 R_{3}, \frac{\overline{R_{4}}}{\gamma}\right\}$ and $\Omega_{4}=\left\{(u, v) \in Y:\|(u, v)\|_{Y}<R_{4}\right\}$. For any $(u, v) \in P \cap \partial \Omega_{4}$, we have $\min _{t \in\left[\theta_{1}, \theta_{2}\right]}(u(t)+v(t)) \geq \gamma\|(u, v)\|_{Y}=\gamma R_{4} \geq \overline{R_{4}}$, then

$$
\begin{aligned}
T_{2}(u, v)\left(\theta_{2}\right) & =\varphi_{q_{2}}(\mu) \int_{0}^{1} G_{2}\left(\theta_{2}, s\right) \varphi_{q_{2}}\left(\int_{0}^{1} H_{2}(s, \tau) g(\tau, u(\tau), v(\tau)) d \tau\right) d s \\
& \geq \varphi_{q_{2}}(\mu) \int_{0}^{1} \frac{\beta_{2}-2}{\Gamma\left(\beta_{2}\right)} \gamma_{2} l_{2}(s) \varphi_{q_{2}}\left(\int_{\theta_{1}}^{\theta_{2}} H_{2}(s, \tau) g(\tau, u(\tau), v(\tau)) d \tau\right) d s \\
& \geq \frac{\beta_{2}-2}{\Gamma\left(\beta_{2}\right)} \gamma_{2} \varphi_{q_{2}}(\mu) \int_{0}^{1} l_{2}(s) \varphi_{q_{2}}\left(\int_{\theta_{1}}^{\theta_{2}} H_{2}(s, \tau) \frac{1}{\varepsilon} \varphi_{p_{2}}(u(\tau)+v(\tau)) d \tau\right) d s \\
& \geq \frac{\beta_{2}-2}{\Gamma\left(\beta_{2}\right)} \gamma_{2} \varphi_{q_{2}}(\mu) \int_{\theta_{1}}^{\theta_{2}} l_{2}(s) \varphi_{q_{2}}\left(\int_{\theta_{1}}^{\theta_{2}} H_{2}(s, \tau) \frac{1}{\varepsilon} \varphi_{p_{2}}\left(\gamma\|(u, v)\|_{Y}\right) d \tau\right) d s \\
& =\frac{\beta_{2}-2}{\Gamma\left(\beta_{2}\right)} \gamma_{2} \gamma \varphi_{q_{2}}\left(\frac{\mu}{\varepsilon}\right) D\|(u, v)\|_{Y}>\|(u, v)\|_{Y} .
\end{aligned}
$$

Therefore

$$
\|Q(u, v)\|_{Y} \geq\left\|T_{2}(u, v)\right\| \geq\|(u, v)\|_{Y}, \quad(u, v) \in P \cap \partial \Omega_{4} .
$$

By (3.4), (3.5) and Lemma 2.8, we conclude that $Q$ has at least one fixed point $(u, v) \in$ $P \cap\left(\overline{\Omega_{4}} \backslash \Omega_{3}\right)$ with $R_{3} \leq\|(u, v)\|_{Y} \leq R_{4}$. This completes the proof.

For $f_{0}^{i}, g_{0}^{i}, f_{\infty}^{s}, g_{\infty}^{s} \in(0, \infty)$, we define the symbols $\widetilde{L_{1}}, \widetilde{L_{2}}, \widetilde{L_{3}}, \widetilde{L_{4}}$ as follows:

$$
\begin{array}{ll}
\tilde{L_{1}}=\varphi_{p_{1}}\left(\frac{\Gamma\left(\beta_{1}\right)}{2\left(\beta_{1}-2\right) C \gamma_{1} \gamma}\right) \frac{1}{f_{0}^{i}}, & \widetilde{L_{2}}=\varphi_{p_{1}}\left(\frac{\Gamma\left(\beta_{1}\right)}{2 A M_{1}}\right) \frac{1}{f_{\infty}^{s}}, \\
\widetilde{L_{3}}=\varphi_{p_{2}}\left(\frac{\Gamma\left(\beta_{2}\right)}{2\left(\beta_{2}-2\right) D \gamma_{2} \gamma}\right) \frac{1}{g_{0}^{i}}, & \widetilde{L_{4}}=\varphi_{p_{2}}\left(\frac{\Gamma\left(\beta_{2}\right)}{2 B M_{2}}\right) \frac{1}{g_{\infty}^{s}} .
\end{array}
$$

Theorem 3.2 (1) If $f_{\infty}^{s}, g_{\infty}^{s}, f_{0}^{i}, g_{0}^{i} \in(0, \infty)$, and $\widetilde{L_{1}}<\widetilde{L_{2}}, \widetilde{L_{3}}<\widetilde{L_{4}}$, then for each $\lambda \in\left(\widetilde{L_{1}}, \widetilde{L_{2}}\right)$ and $\mu \in\left(\widetilde{L_{3}}, \widetilde{L_{4}}\right)$, system $(1.1)$ has at least one positive solution $(u(t), v(t)), t \in(0,1)$.

(2) If $f_{\infty}^{s}, f_{0}^{i}, g_{0}^{i} \in(0, \infty), g_{\infty}^{s}=0$, and $\widetilde{L_{1}}<\widetilde{L_{2}}$, then for each $\lambda \in\left(\widetilde{L_{1}}, \widetilde{L_{2}}\right)$ and $\mu \in\left(\widetilde{L_{3}}, \infty\right)$, system (1.1) has at least one positive solution $(u(t), v(t)), t \in(0,1)$.

(3) If $g_{\infty}^{s}, f_{0}^{i}, g_{0}^{i} \in(0, \infty), f_{\infty}^{s}=0, \widetilde{L_{3}}<\widetilde{L_{4}}$, then for each $\lambda \in\left(\widetilde{L_{1}}, \infty\right)$ and $\mu \in\left(\widetilde{L_{3}}, \widetilde{L_{4}}\right)$, system (1.1) has at least one positive solution $(u(t), v(t)), t \in(0,1)$.

(4) If $f_{0}^{i}, g_{0}^{i} \in(0, \infty), f_{\infty}^{s}=g_{\infty}^{s}=0$, then for each $\lambda \in\left(\widetilde{L_{1}}, \infty\right)$ and $\mu \in\left(\widetilde{L_{3}}, \infty\right)$, system $(1.1)$ has at least one positive solution $(u(t), v(t)), t \in(0,1)$.

(5) If $\left\{f_{\infty}^{s}, g_{\infty}^{s} \in(0, \infty), f_{0}^{i}=\infty\right\}$ or $\left\{f_{\infty}^{s}, g_{\infty}^{s} \in(0, \infty), g_{0}^{i}=\infty\right\}$, then for each $\lambda \in\left(0, \widetilde{L_{2}}\right)$ and $\mu \in\left(0, \widetilde{L_{4}}\right)$, system (1.1) has at least one positive solution $(u(t), v(t)), t \in(0,1)$. 
(6) If $\left\{f_{0}^{i}=\infty, f_{\infty}^{s} \in(0, \infty), g_{\infty}^{s}=0\right\}$ or $\left\{f_{\infty}^{s} \in(0, \infty), g_{\infty}^{s}=0, g_{0}^{i}=\infty\right\}$, then for each $\lambda \in$ $\left(0, \widetilde{L_{2}}\right)$ and $\mu \in(0, \infty)$, system (1.1) has at least one positive solution $(u(t), v(t)), t \in(0,1)$.

(7) If $\left\{f_{0}^{i}=\infty, g_{\infty}^{s} \in(0, \infty), f_{\infty}^{s}=0\right\}$ or $\left\{g_{\infty}^{s} \in(0, \infty), g_{0}^{i}=\infty, f_{\infty}^{s}=0\right\}$, then for each $\lambda \in$ $(0, \infty)$ and $\mu \in\left(0, \widetilde{L_{4}}\right)$, system (1.1) has at least one positive solution $(u(t), v(t)), t \in(0,1)$.

(8) If $\left\{f_{\infty}^{s}=g_{\infty}^{s}=0, f_{0}^{i}=\infty\right\}$ or $\left\{f_{\infty}^{s}=g_{\infty}^{s}=0, g_{0}^{i}=\infty\right\}$, then for each $\lambda \in(0, \infty)$ and $\mu \in$ $(0, \infty)$, system (1.1) has at least one positive solution $(u(t), v(t)), t \in(0,1)$.

Proof Because the proofs of the above cases are similar, in what follows we will prove two of them, namely cases (1) and (6).

(1) For any $\lambda \in\left(\widetilde{L_{1}}, \widetilde{L_{2}}\right)$ and $\mu \in\left(\widetilde{L_{3}}, \widetilde{L_{4}}\right)$, there exists $0<\varepsilon<\min \left\{f_{0}^{i}, g_{0}^{i}\right\}$ such that

$$
\begin{aligned}
& \varphi_{p_{1}}\left(\frac{\Gamma\left(\beta_{1}\right)}{2\left(\beta_{1}-2\right) C \gamma_{1} \gamma}\right) \frac{1}{f_{0}^{i}-\varepsilon} \leq \lambda \leq \varphi_{p_{1}}\left(\frac{\Gamma\left(\beta_{1}\right)}{2 A M_{1}}\right) \frac{1}{f_{\infty}^{s}+\varepsilon}, \\
& \varphi_{p_{2}}\left(\frac{\Gamma\left(\beta_{2}\right)}{2\left(\beta_{2}-2\right) D \gamma_{2} \gamma}\right) \frac{1}{g_{0}^{i}-\varepsilon} \leq \mu \leq \varphi_{p_{2}}\left(\frac{\Gamma\left(\beta_{2}\right)}{2 B M_{2}}\right) \frac{1}{g_{\infty}^{s}+\varepsilon} .
\end{aligned}
$$

By the definitions of $f_{0}^{i}$ and $g_{0}^{i}$, there exists $R_{1}>0$ such that

$$
\begin{aligned}
& f(t, u, v) \geq\left(f_{0}^{i}-\varepsilon\right) \varphi_{p_{1}}(u+v), \quad t \in\left[\theta_{1}, \theta_{2}\right], u, v \geq 0, u+v \leq R_{1}, \\
& g(t, u, v) \geq\left(g_{0}^{i}-\varepsilon\right) \varphi_{p_{2}}(u+v), \quad t \in\left[\theta_{1}, \theta_{2}\right], u, v \geq 0, u+v \leq R_{1} .
\end{aligned}
$$

Denote $\Omega_{1}=\left\{(u, v) \in Y:\|(u, v)\|<R_{1}\right\}$, for any $(u, v) \in P \cap \partial \Omega_{1}$, we have

$$
\begin{aligned}
& T_{1}(u, v)\left(\theta_{1}\right) \\
& \quad=\varphi_{q_{1}}(\lambda) \int_{0}^{1} G_{1}\left(\theta_{1}, s\right) \varphi_{q_{1}}\left(\int_{0}^{1} H_{1}(s, \tau) f(\tau, u(\tau), v(\tau)) d \tau\right) d s \\
& \quad \geq \varphi_{q_{1}}(\lambda) \int_{\theta_{1}}^{\theta_{2}} \frac{\beta_{1}-2}{\Gamma\left(\beta_{1}\right)} \gamma_{1} l_{1}(s) \varphi_{q_{1}}\left(\int_{\theta_{1}}^{\theta_{2}} H_{1}(s, \tau) f(\tau, u(\tau), v(\tau)) d \tau\right) d s \\
& \quad \geq \frac{\beta_{1}-2}{\Gamma\left(\beta_{1}\right)} \gamma_{1} \varphi_{q_{1}}(\lambda) \int_{\theta_{1}}^{\theta_{2}} l_{1}(s) \varphi_{q_{1}}\left(\int_{\theta_{1}}^{\theta_{2}} H_{1}(s, \tau)\left(f_{0}^{i}-\varepsilon\right) \varphi_{p_{1}}(u(\tau)+v(\tau)) d \tau\right) d s \\
& \quad \geq \frac{\beta_{1}-2}{\Gamma\left(\beta_{1}\right)} \gamma_{1} \gamma \varphi_{q_{1}}\left(\lambda\left(f_{0}^{i}-\varepsilon\right)\right) C\|(u, v)\|_{Y} \geq \frac{1}{2}\|(u, v)\|_{Y},
\end{aligned}
$$

then $\left\|T_{1}(u, v)\right\| \geq \frac{1}{2}\|(u, v)\|_{Y},(u, v) \in P \cap \partial \Omega_{1}$. Similarly, we have

$$
\begin{aligned}
& T_{2}(u, v)\left(\theta_{2}\right) \\
& \quad=\varphi_{q_{2}}(\mu) \int_{0}^{1} G_{2}\left(\theta_{2}, s\right) \varphi_{q_{2}}\left(\int_{0}^{1} H_{2}(s, \tau) g(\tau, u(\tau), v(\tau)) d \tau\right) d s \\
& \quad \geq \varphi_{q_{2}}(\mu) \int_{\theta_{1}}^{\theta_{2}} \frac{\beta_{2}-2}{\Gamma\left(\beta_{2}\right)} \gamma_{2} l_{2}(s) \varphi_{q_{2}}\left(\int_{\theta_{1}}^{\theta_{2}} H_{2}(s, \tau) g(\tau, u(\tau), v(\tau)) d \tau\right) d s \\
& \quad \geq \frac{\beta_{2}-2}{\Gamma\left(\beta_{2}\right)} \gamma_{2} \varphi_{q_{2}}(\mu) \int_{\theta_{1}}^{\theta_{2}} l_{2}(s) \varphi_{q_{2}}\left(\int_{\theta_{1}}^{\theta_{2}} H_{2}(s, \tau)\left(g_{0}^{i}-\varepsilon\right) \varphi_{p_{2}}(u(\tau)+v(\tau)) d \tau\right) d s \\
& \quad \geq \frac{\beta_{2}-2}{\Gamma\left(\beta_{2}\right)} \gamma_{2} \gamma \varphi_{q_{2}}\left(\mu\left(g_{0}^{i}-\varepsilon\right)\right) D\|(u, v)\|_{Y} \geq \frac{1}{2}\|(u, v)\|_{Y},
\end{aligned}
$$


then $\left\|T_{2}(u, v)\right\| \geq \frac{1}{2}\|(u, v)\|_{Y},(u, v) \in P \cap \partial \Omega_{1}$. Therefore

$$
\|Q(u, v)\|_{Y} \geq\|(u, v)\|_{Y}, \quad(u, v) \in P \cap \partial \Omega_{1} .
$$

On the other hand, we define $f^{*}, g^{*}:[0,1] \times[0,+\infty) \rightarrow[0,+\infty)$ as follows:

$$
f^{*}(t, x)=\max _{0 \leq u+v \leq x} f(t, u, v), \quad g^{*}(t, x)=\max _{0 \leq u+v \leq x} g(t, u, v)
$$

then

$$
\begin{aligned}
& f(t, u, v) \leq f^{*}(t, x), \quad t \in[0,1], u, v \geq 0, u+v \leq x, \\
& g(t, u, v) \leq g^{*}(t, x), \quad t \in[0,1], u, v \geq 0, u+v \leq x .
\end{aligned}
$$

Clearly, $f^{*}(t, x)$ and $g^{*}(t, x)$ are nondecreasing on $x$, by the proof of [35], we have

$$
\limsup _{x \rightarrow+\infty} \max _{t \in[0,1]} \frac{f^{*}(t, x)}{\varphi_{p_{1}}(x)} \leq f_{\infty}^{s}, \quad \limsup _{x \rightarrow+\infty} \max _{t \in[0,1]} \frac{g^{*}(t, x)}{\varphi_{p_{2}}(x)} \leq g_{\infty}^{s} .
$$

From the above inequalities, there exists $\overline{R_{2}}>0$ such that

$$
\begin{aligned}
& \frac{f^{*}(t, x)}{\varphi_{p_{1}}(x)} \leq \limsup _{x \rightarrow+\infty} \max _{t \in[0,1]} \frac{f^{*}(t, x)}{\varphi_{p_{1}}(x)}+\varepsilon \leq f_{\infty}^{s}+\varepsilon, \quad t \in[0,1], x \geq \overline{R_{2}}, \\
& \frac{g^{*}(t, x)}{\varphi_{p_{2}}(x)} \leq \limsup _{x \rightarrow+\infty} \max _{t \in[0,1]} \frac{g^{*}(t, x)}{\varphi_{p_{2}}(x)}+\varepsilon \leq g_{\infty}^{s}+\varepsilon, \quad t \in[0,1], x \geq \overline{R_{2}} .
\end{aligned}
$$

Then $f^{*}(t, x) \leq\left(f_{\infty}^{s}+\varepsilon\right) \varphi_{p_{1}}(x), g^{*}(t, x) \leq\left(g_{\infty}^{s}+\varepsilon\right) \varphi_{p_{2}}(x), t \in[0,1], x \geq \overline{R_{2}}$.

Denote $R_{2}=\max \left\{2 R_{1}, \overline{R_{2}}\right\}, \Omega_{2}=\left\{(u, v) \in Y:\|(u, v)\|_{Y}<R_{2}\right\}$. For any $(u, v) \in P \cap \partial \Omega_{2}$, by the definitions of $f^{*}$ and $g^{*}$, we have

$$
f(t, u(t), v(t)) \leq f^{*}\left(t,\|(u, v)\|_{Y}\right), \quad g(t, u(t), v(t)) \leq g^{*}\left(t,\|(u, v)\|_{Y}\right), \quad t \in[0,1]
$$

so

$$
\begin{aligned}
T_{1}(u, v)(t) & \leq \varphi_{q_{1}}(\lambda) \int_{0}^{1} \frac{M_{1}}{\Gamma\left(\beta_{1}\right)} l_{1}(s) \varphi_{q_{1}}\left(\int_{0}^{1} H_{1}(s, \tau) f^{*}\left(\tau,\|(u, v)\|_{Y}\right) d \tau\right) d s \\
& \leq \varphi_{q_{1}}(\lambda) \frac{M_{1}}{\Gamma\left(\beta_{1}\right)} \int_{0}^{1} l_{1}(s) \varphi_{q_{1}}\left(\int_{0}^{1} H_{1}(s, \tau)\left(f_{\infty}^{s}+\varepsilon\right) \varphi_{p_{1}}\left(\|(u, v)\|_{Y}\right) d \tau\right) d s \\
& =\frac{M_{1}}{\Gamma\left(\beta_{1}\right)} \varphi_{q_{1}}\left(\lambda\left(f_{\infty}^{s}+\varepsilon\right)\right) A\|(u, v)\|_{Y} \leq \frac{1}{2}\|(u, v)\|_{Y},
\end{aligned}
$$

and $\left\|T_{1}(u, v)\right\| \leq \frac{1}{2}\|(u, v)\|_{Y},(u, v) \in P \cap \partial \Omega_{2}$. Similarly, we have

$$
\begin{aligned}
T_{2}(u, v)(t) & \leq \varphi_{q_{2}}(\mu) \int_{0}^{1} \frac{M_{2}}{\Gamma\left(\beta_{2}\right)} l_{2}(s) \varphi_{q_{2}}\left(\int_{0}^{1} H_{2}(s, \tau) g^{*}\left(\tau,\|(u, v)\|_{Y}\right) d \tau\right) d s \\
& \leq \varphi_{q_{2}}(\mu) \frac{M_{2}}{\Gamma\left(\beta_{2}\right)} \int_{0}^{1} l_{2}(s) \varphi_{q_{2}}\left(\int_{0}^{1} H_{2}(s, \tau)\left(g_{\infty}^{s}+\varepsilon\right) \varphi_{p_{2}}\left(\|(u, v)\|_{Y}\right) d \tau\right) d s \\
& =\frac{M_{2}}{\Gamma\left(\beta_{2}\right)} \varphi_{q_{2}}\left(\mu\left(g_{\infty}^{s}+\varepsilon\right)\right) B\|(u, v)\|_{Y} \leq \frac{1}{2}\|(u, v)\|_{Y},
\end{aligned}
$$


so $\left\|T_{2}(u, v)\right\| \leq \frac{1}{2}\|(u, v)\|_{Y},(u, v) \in P \cap \partial \Omega_{2}$. Therefore

$$
\|Q(u, v)\|_{Y}=\left\|T_{1}(u, v)\right\|+\left\|T_{2}(u, v)\right\| \leq\|(u, v)\|_{Y}, \quad(u, v) \in P \cap \partial \Omega_{2} .
$$

From (3.6), (3.7) and Lemma 2.8, we get that $Q$ has at least one fixed point $(u, v) \in$ $P \cap\left(\overline{\Omega_{2}} \backslash \Omega_{1}\right)$ with $R_{1} \leq\|(u, v)\|_{Y} \leq R_{2}$.

(6) Suppose $f_{0}^{i}=\infty, f_{\infty}^{s} \in(0, \infty), g_{\infty}^{s}=0$, for any $\lambda \in\left(0, \widetilde{L_{2}}\right)$ and $\mu \in(0, \infty)$, there exists $\varepsilon>0$ such that

$$
\varphi_{p_{1}}\left(\frac{\Gamma\left(\beta_{1}\right)}{\left(\beta_{1}-2\right) C \gamma_{1} \gamma}\right) \varepsilon<\lambda<\varphi_{p_{1}}\left(\frac{\Gamma\left(\beta_{1}\right)}{2 A M_{1}}\right) \frac{1}{f_{\infty}^{0}+\varepsilon}, \quad 0<\mu<\varphi_{p_{2}}\left(\frac{\Gamma\left(\beta_{2}\right)}{2 B M_{2}}\right) \frac{1}{\varepsilon} .
$$

By $f_{0}^{i}=\infty$, there exists $R_{3}>0$ such that

$$
f(t, u, v) \geq \frac{1}{\varepsilon} \varphi_{p_{1}}(u+v), \quad t \in\left[\theta_{1}, \theta_{2}\right], u, v \geq 0,0 \leq u+v \leq R_{3} .
$$

Choose $\Omega_{3}=\left\{(u, v) \in Y:\|(u, v)\|_{Y}<R_{3}\right\}$, then for any $(u, v) \in P \cap \partial \Omega_{3}$, we have

$$
\begin{aligned}
T_{1}(u, v)\left(\theta_{1}\right) & \geq \varphi_{q_{1}}(\lambda) \int_{\theta_{1}}^{\theta_{2}} \frac{\beta_{1}-2}{\Gamma\left(\beta_{1}\right)} \gamma_{1} l_{1}(s) \varphi_{q_{1}}\left(\int_{\theta_{1}}^{\theta_{2}} H_{1}(s, \tau) f(\tau, u(\tau), v(\tau)) d \tau\right) d s \\
& \geq \frac{\beta_{1}-2}{\Gamma\left(\beta_{1}\right)} \gamma_{1} \varphi_{q_{1}}(\lambda) \int_{\theta_{1}}^{\theta_{2}} l_{1}(s) \varphi_{q_{1}}\left(\int_{\theta_{1}}^{\theta_{2}} H_{1}(s, \tau) \frac{1}{\varepsilon} \varphi_{p_{1}}(u(\tau)+v(\tau)) d \tau\right) d s \\
& \geq \frac{\beta_{1}-2}{\Gamma\left(\beta_{1}\right)} \gamma_{1} \gamma \varphi_{q_{1}}\left(\frac{\lambda}{\varepsilon}\right) \int_{\theta_{1}}^{\theta_{2}} l_{1}(s) \varphi_{q_{1}}\left(\int_{\theta_{1}}^{\theta_{2}} H_{1}(s, \tau) d \tau\right) d s\|(u, v)\|_{Y} \\
& =\frac{\beta_{1}-2}{\Gamma\left(\beta_{1}\right)} \gamma_{1} \gamma \varphi_{q_{1}}\left(\frac{\lambda}{\varepsilon}\right) C\|(u, v)\|_{Y} \geq\|(u, v)\|_{Y} .
\end{aligned}
$$

Thus,

$$
\|Q(u, v)\|_{Y} \geq\left\|T_{1}(u, v)\right\| \geq\|(u, v)\|_{Y}, \quad(u, v) \in P \cap \partial \Omega_{3} .
$$

On the other hand, we define $f^{*}, g^{*}:[0,1] \times[0,+\infty) \rightarrow[0,+\infty)$ as follows:

$$
f^{*}(t, x)=\max _{0 \leq u+v \leq x} f(t, u, v), \quad g^{*}(t, x)=\max _{0 \leq u+v \leq x} g(t, u, v) .
$$

By the proof of [35], we have

$$
\limsup _{x \rightarrow+\infty} \max _{t \in[0,1]} \frac{f^{*}(t, x)}{\varphi_{p_{1}}(x)} \leq f_{\infty}^{s}, \quad \lim _{x \rightarrow+\infty} \max _{t \in[0,1]} \frac{g^{*}(t, x)}{\varphi_{p_{2}}(x)}=0 .
$$

For above $\varepsilon>0$, there exists $\overline{R_{4}}>0$ such that, for any $t \in[0,1], x \geq \overline{R_{4}}$, we have

$$
\begin{aligned}
& \frac{f^{*}(t, x)}{\varphi_{p_{1}}(x)} \leq \limsup _{x \rightarrow+\infty} \max _{t \in[0,1]} \frac{f^{*}(t, x)}{\varphi_{p_{1}}(x)}+\varepsilon \leq f_{\infty}^{s}+\varepsilon, \\
& \frac{g^{*}(t, x)}{\varphi_{p_{2}}(x)} \leq \lim _{x \rightarrow+\infty} \max _{t \in[0,1]} \frac{g^{*}(t, x)}{\varphi_{p_{2}}(x)}+\varepsilon=\varepsilon,
\end{aligned}
$$

hence $f^{*}(t, x) \leq\left(f_{\infty}^{s}+\varepsilon\right) \varphi_{p_{1}}(x), g^{*}(t, x) \leq \varepsilon \varphi_{p_{2}}(x)$. 
Let $R_{4}=\max \left\{2 R_{3}, \overline{R_{4}}\right\}$ and $\Omega_{4}=\left\{(u, v) \in Y:\|(u, v)\|_{Y}<R_{4}\right\}$. For any $(u, v) \in P \cap \partial \Omega_{4}$ and $t \in[0,1]$, we have

$$
f(t, u(t), v(t)) \leq f^{*}\left(t,\|(u, v)\|_{Y}\right), \quad g(t, u(t), v(t)) \leq g^{*}\left(t,\|(u, v)\|_{Y}\right),
$$

then

$$
\begin{aligned}
T_{1}(u, v)(t) & \leq \varphi_{q_{1}}(\lambda) \int_{0}^{1} \frac{M_{1}}{\Gamma\left(\beta_{1}\right)} l_{1}(s) \varphi_{q_{1}}\left(\int_{0}^{1} H_{1}(s, \tau) f^{*}\left(\tau,\|(u, v)\|_{Y}\right) d \tau\right) d s \\
& \leq \varphi_{q_{1}}(\lambda) \frac{M_{1}}{\Gamma\left(\beta_{1}\right)} \int_{0}^{1} l_{1}(s) \varphi_{q_{1}}\left(\int_{0}^{1} H_{1}(s, \tau)\left(f_{\infty}^{s}+\varepsilon\right) \varphi_{p_{1}}\left(\|(u, v)\|_{Y}\right) d \tau\right) d s \\
& =\frac{M_{1}}{\Gamma\left(\beta_{1}\right)} \varphi_{q_{1}}\left(\lambda\left(f_{\infty}^{s}+\varepsilon\right)\right) A\|(u, v)\|_{Y} \leq \frac{1}{2}\|(u, v)\|_{Y},
\end{aligned}
$$

so $\left\|T_{1}(u, v)\right\| \leq \frac{1}{2}\|(u, v)\|_{Y},(u, v) \in P \cap \partial \Omega_{4}$. In a similar manner, we deduce

$$
\begin{aligned}
T_{2}(u, v)(t) & \leq \varphi_{q_{2}}(\mu) \int_{0}^{1} \frac{M_{2}}{\Gamma\left(\beta_{2}\right)} l_{2}(s) \varphi_{q_{2}}\left(\int_{0}^{1} H_{2}(s, \tau) g^{*}\left(\tau,\|(u, v)\|_{Y}\right) d \tau\right) d s \\
& \leq \varphi_{q_{2}}(\mu) \frac{M_{2}}{\Gamma\left(\beta_{2}\right)} \int_{0}^{1} l_{2}(s) \varphi_{q_{2}}\left(\int_{0}^{1} H_{2}(s, \tau) \varepsilon \varphi_{p_{2}}\left(\|(u, v)\|_{Y}\right) d \tau\right) d s \\
& =\frac{M_{2}}{\Gamma\left(\beta_{2}\right)} \varphi_{q_{2}}(\mu \varepsilon) B\|(u, v)\|_{Y} \leq \frac{1}{2}\|(u, v)\|_{Y},
\end{aligned}
$$

so $\left\|T_{2}(u, v)\right\| \leq \frac{1}{2}\|(u, v)\|_{Y},(u, v) \in P \cap \partial \Omega_{4}$. Therefore

$$
\|Q(u, v)\|_{Y}=\left\|T_{1}(u, v)\right\|+\left\|T_{2}(u, v)\right\| \leq\|(u, v)\|_{Y}, \quad(u, v) \in P \cap \partial \Omega_{4} .
$$

From (3.8), (3.9) and Lemma 2.8, we conclude that $Q$ has at least one fixed point $(u, v) \in$ $P \cap\left(\overline{\Omega_{4}} \backslash \Omega_{3}\right)$ with $R_{3} \leq\|(u, v)\|_{Y} \leq R_{4}$. This completes the proof.

\section{Conclusion}

In this paper, we study the existence of positive solutions for a system of nonlinear fractional differential equations nonlocal boundary value problems with parameters and $p$ Laplacian operator. Under different combinations of superlinearity and sublinearity of the functions $f$ and $g$, various existence results for positive solutions are derived in terms of different values of $\lambda$ and $\mu$ via the Guo-Krasnosel'skii fixed point theorem.

\section{Acknowledgements}

The authors would like to thank the referees for their pertinent comments and valuable suggestions.

\section{Funding}

This work is supported financially by the National Natural Science Foundation of China $(11501318,11371221)$, the Natural Science Foundation of Shandong Province of China (ZR2015AM022, ZR2017MA036) and the China Postdoctoral Science Foundation (2017M612230).

\section{Abbreviations}

Not applicable.

Availability of data and materials 


\section{Competing interests}

The authors declare that they have no competing interests.

\section{Authors' contributions}

All authors contributed equally to the writing of this paper. The authors read and approved the final manuscript.

\section{Author details}

'School of Mathematical Sciences, Qufu Normal University, Qufu, Shandong 273165, P.R. China. ${ }^{2}$ Department of Mathematics and Statistics, Curtin University, Perth, WA 6845, Australia. ${ }^{3}$ Department of Applied Mathematics, Shandong University of Science and Technology, Qingdao, 266590, P.R. China.

\section{Publisher's Note}

Springer Nature remains neutral with regard to jurisdictional claims in published maps and institutional affiliations.

Received: 24 October 2017 Accepted: 1 December 2017 Published online: 11 December 2017

\section{References}

1. Podlubny, I: Fractional Differential Equations. Academic Press, New York (1999)

2. Kilbas, AA, Srivastava, HM, Trujillo, JJ: Theory and Applications of Fractional Differential Equations. Elsevier, Amsterdam (2006)

3. Miller, KS, Ross, B: An Introduction to the Fractional Calculus and Fractional Differential Equations. Wiley, New York (1993)

4. Leibenson, LS: General problem of the movement of a compressible fluid in a porous medium. Izvestiia Akad. Nauk SSSR 9, 7-10 (1945)

5. Liu, X, Jia, M, Ge, W: The method of lower and upper solutions for mixed fractional four-point boundary value problem with p-Laplacian operator. Appl. Math. Lett. 65, 56-62 (2017)

6. Jiang, W, Qiu, J, Yang, C: The existence of solutions for fractional differential equations with $p$-Laplacian at resonance. Chaos 27, 032102 (2017)

7. Dong, X, Bai, Z, Zhang, S: Positive solutions to boundary value problems of $p$-Laplacian with fractional derivative. Bound. Value Probl. 2017, 5 (2017)

8. Chen, T, Liu, W, Liu, J: Solvability of periodic boundary value problem for fractional $p$-Laplacian equation. Appl. Math. Comput. 24, 422-431 (2014)

9. Liu, X, Jia, M, Xiang, X: On the solvability of a fractional differential equation model involving the $p$-Laplacian operator. Comput. Math. Appl. 64, 3267-3275 (2012)

10. Ding, Y, Wei, Z: On the extremal solution for a nonlinear boundary value problems of fractional $p$-Laplacian operator. Filomat 30, 3771-3778 (2016)

11. Jafari, $H$, Baleanu, $D$, Khan, $H$, Akhan, $R$, Khan, A: Existence criterion for the solutions of fractional order $p$-Laplacian boundary value problems. Bound. Value Probl. 2015, 164 (2015)

12. Wang, $Y$, Liu, L, Wu, Y: Extremal solutions for $p$-Laplacian fractional integro-differential equation with integral conditions on infinite integrals via iterative computation. Adv. Differ. Equ. 2015, 24 (2015)

13. Guo, X: Existence of unique solution to switched fractional differential equations with $p$-Laplacian operator. Turk. J. Math. 39, 864-871 (2015)

14. Liang, S, Zhang, J: Existence and uniqueness of positive solutions for integral boundary problems of nonlinear fractional differential equations with p-Laplacian operator. Rocky Mt. J. Math. 44, 953-974 (2014)

15. Lu, H, Han, Z, Sun, S, Liu, J: Existence on positive solution for boundary value problems of nonlinear fractional differential equations with p-Laplacian. Adv. Differ. Equ. 2013, 30 (2013)

16. Shen, T, Liu, W, Shen, X: Existence and uniqueness of solutions for several BVPs of fractional differential equations with p-Laplacian operator. Mediterr. J. Math. 13, 4623-4637 (2016)

17. $\mathrm{Xu}, \mathrm{J}$, Dong, W: Existence and uniqueness of positive solutions for a fractional boundary value problem with p-Laplacian operator. Acta Math. Sin. (Chin. Ser.) 59, 385-396 (2016)

18. Zhang, $\mathrm{X}$, Liu, L, Wiwatanapataphee, $\mathrm{B}, \mathrm{Wu}, \mathrm{Y}$ : The eigenvalue for a class of singular $p$-Laplacian fractional differential equations involving the Riemann-Stieltjes integral boundary condition. Appl. Math. Comput. 235, 412-422 (2014)

19. Wu, J, Zhang, X, Liu, L, Wu, Y: Twin iterative solutions for a fractional differential turbulent flow model. Bound. Value Probl. 2016, 98 (2016)

20. Lv, Z: Existence results for $m$-point boundary value problems of nonlinear fractional differential equations with p-Laplacian operator. Adv. Differ. Equ. 2014, 69 (2014)

21. LV, Z: Existence results of fractional differential equations with irregular boundary conditions and $p$-Laplacian operator. J. Appl. Math. Comput. 46, 33-49 (2014)

22. Liu, Z, Lu, L: A class of BVPs for nonlinear fractional differential equations with $p$-Laplacian operator. Electron. J. Qual. Theory Differ. Equ. 2012, 70 (2012)

23. Tan, J, Cheng, C: Existence of solutions of boundary value problems for fractional differential equations with p-Laplacian operator in Banach spaces. Numer. Funct. Anal. Optim. 38, 738-753 (2017)

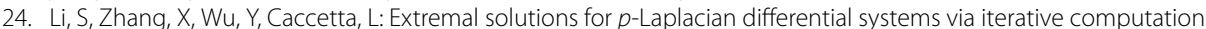
Appl. Math. Lett. 26, 1151-1158 (2013)

25. Ren, T, Li, S, Zhang, X, Liu, L: Maximum and minimum solutions for a nonlocal $p$-Laplacian fractional differentia system from eco-economical processes. Bound. Value Probl. 2017, 118 (2017)

26. Rao, SN: Multiplicity of positive solutions for coupled system of fractional differential equation with $p$-Laplacian two-point BVPs. J. Appl. Math. Comput. 55, 41-58 (2017)

27. Liu, Y, Xie, D, Bai, C, Yang, D: Multiple positive solutions for a coupled system of fractional multi-point BVP with p-Laplacian operator. Adv. Differ. Equ. 2017, 168 (2017) 
28. $\mathrm{Hu}, \mathrm{L}, \mathrm{Zhang}, \mathrm{S}$ : Existence results for a coupled system of fractional differential equations with $p$-Laplacian operator and infinite-point boundary conditions. Bound. Value Probl. 2017, 88 (2017)

29. He, J, Song, $X$ : The uniqueness of solution for a class of fractional order nonlinear systems with $p$-Laplacian operator. Abstr. Appl. Anal. 2014, Article ID 921209 (2014)

30. He, Y: The eigenvalue problem for a coupled system of singular $p$-Laplacian differential equations involving fractional differential-integral conditions. Adv. Differ. Equ. 2016, 209 (2016)

31. Khan, A, Li, Y, Shah, K, Khan, TS: On coupled $p$-Laplacian fractional differential equations with nonlinear boundary conditions. Complexity 2017, Article ID 8197610 (2017)

32. Cheng, L, Liu, W, Ye, Q: Boundary value problem for a coupled system of fractional differential equations with p-Laplacian operator at resonance. Electron. J. Differ. Equ. 2014, 60 (2014)

33. Xu, X, Jiang, D, Yuan, C: Multiple positive solutions for the boundary value problem of a nonlinear fractional differential equation. Nonlinear Anal. 71, 4676-4688 (2009)

34. Guo, D, Lakshmikantham, V: Nonlinear Problems in Abstract Cones. Academic Press, New York (1988)

35. Wang, H: On the number of positive solutions of nonlinear systems. J. Math. Anal. Appl. 281, 287-306 (2003)

\section{Submit your manuscript to a SpringerOpen ${ }^{\circ}$ journal and benefit from:}

- Convenient online submission

- Rigorous peer review

Open access: articles freely available online

- High visibility within the field

- Retaining the copyright to your article

Submit your next manuscript at $\boldsymbol{\nabla}$ springeropen.com 